\title{
Treatment Outcomes of Venlafaxine and Duloxetine in Refractory Burning Mouth Syndrome Patients
}

\author{
Moon-Jong Kim ${ }^{1}$, Hong-Seop Kho ${ }^{1,2}$ \\ 'Department of Oral Medicine and Oral Diagnosis, School of Dentistry and Dental Research Institute, \\ Seoul National University, Seoul, Korea \\ ${ }^{2}$ Institute on Aging Seoul National University, Seoul, Korea
}

Received July 31, 2019

Revised August 30, 2019

Accepted August 30, 2019

\begin{abstract}
Correspondence to:
Hong-Seop Kho

Department of Oral Medicine and Oral

Diagnosis, School of Dentistry and Dental

Research Institute, Seoul National University,

101 Daehak-ro, Jongno-gu, Seoul 03080,

Korea

Tel: +82-2-2072-3989

Fax: +82-2-744-9135

E-mail: hkho@snu.ac.kr

https://orcid.org/0000-0001-9960-9892
\end{abstract}

This study was supported by the National Research Foundation of Korea (NRF) grant funded by the Korean government (MSIP) (No. 2016R1A2B4007286).
Purpose: Venlafaxine and duloxetine have been shown to be effective in the treatment of neuropathic pain disorders. However, knowledge about the efficacy of venlafaxine and duloxetine on burning mouth syndrome (BMS) is still insufficient. The purpose of this study was to investigate the efficacy of venlafaxine and duloxetine on refractory BMS patients.

Methods: Twelve refractory BMS patients who were prescribed venlafaxine or duloxetine were included in this study. These patients did not respond to previous administration of clonazepam, alpha-lipoic acid, gabapentin, and nortriptyline. All participants were the primary type of BMS patients who had no local and systemic factors related to the oral burning sensation. The intensities of oral symptoms following venlafaxine or duloxetine administration were compared with those before administration and at baseline.

Results: Venlafaxine and duloxetine were prescribed to four and nine patients, respectively. One patient was prescribed both medications in turn. Among them, only two patients showed improvement of oral symptoms without side effects. In the other ten patients, symptoms failed to improve. Six of them reported that the drug was ineffective, and four of them stopped taking the medications on their own due to intolerable side effects, such as insomnia, constipation, drowsiness, dizziness, and xerostomia.

Conclusions: Venlafaxine and duloxetine may only relieve oral symptoms in a minority of refractory BMS patients. Further large-scale studies are needed to determine the potential clinical factors that could predict the efficacy of venlafaxine and duloxetine.

Key Words: Burning mouth syndrome; Duloxetine; Venlafaxine

\section{INTRODUCTION}

Burning mouth syndrome (BMS) is a chronic orofacial pain disorder defined as persistent burning sensation and other dysesthesia that appears in clinically normal oral mucosa in the absence of abnormal laboratory findings [1,2]. Although the pathophysiology of BMS is complex and various factors are thought to be involved in the development of this condition, BMS is now considered to be a disorder caused by neuropathic changes occurring at various levels of the neuraxis $[3,4]$.

Medications used for neuropathic pain disorders have been applied to intervene in the neuropathic mechanism in BMS patients. Of these medications, clonazepam has been the most widely used medication in BMS management. Clonazepam has been administered topically and orally, and several controlled studies have reported that both administration methods are effective for BMS [5-7]. A recent meta-analysis has also reported that clonazepam is effective in relieving the symptoms of BMS [8]. Alpha-lipoic acid

Copyright (c) 2019 Korean Academy of Orofacial Pain and Oral Medicine. All rights reserved.

(c) This is an open-access article distributed under the terms of the Creative Commons Attribution Non-Commercial License (http://creativecommons.org/licenses/by-nc/4.0/), which permits unrestricted non-commercial use, distribution, and reproduction in any medium, provided the original work is properly cited. 
and gabapentin are also commonly applied to BMS patients [9], and the combination of both medications was more effective than alpha-lipoic acid or gabapentin alone [10]. Tricyclic antidepressants (TCAs), including nortriptyline and amitriptyline have also been used for the treatment of BMS in previous studies [11,12]. However, TCAs have an anticholinergic effect as a side effect which can lead to dry mouth that negatively affects BMS symptoms.

Notwithstanding these medication therapies, only less than half of BMS patients reported significant improvement in their symptoms during long-term follow-up [13,14]. Because dysfunctions at various levels of the peripheral and central nervous systems are involved in the pathogenesis of BMS, there is no consistently effective medication for BMS patients and generally accepted guideline for pharmacotherapy of BMS have not been presented. Thus, the management of BMS remains challenging.

Venlafaxine and duloxetine, serotonin norepinephrine reuptake inhibitors (SNRIs), are known to have a similar efficacy and mechanism of action as TCAs, but do not have an anticholinergic effect. Venlafaxine and duloxetine have also been shown to be effective in chronic pain disorders [15-18]. In a few studies, venlafaxine and duloxetine have been applied to the management of BMS. One study of eight patients with BMS has shown that venlafaxine combined with clonazepam could be an effective treatment [19]. However, no studies have examined the efficacy of venlafaxine alone. Although the beneficial effects of duloxetine on refractory BMS have been documented in two studies [20,21], both studies are single case reports. In addition, another study reported contradictory results that the oral symptoms of one patient who was prescribed duloxetine did not improve [22]. Therefore, knowledge about the efficacy of venlafaxine and duloxetine on BMS is still insufficient. In this study, we reported the efficacy of venlafaxine or duloxetine in 12 refractory BMS patients.

\section{MATERIALS AND METHODS}

\section{Participants}

Twelve refractory BMS patients who were prescribed venlafaxine and duloxetine were included in this study. At the first visit to our clinic, all participants underwent comprehensive clinical examination procedures, and were found to have no local and systemic factors that could cause an oral burning sensation. Various medications, including anticonvulsants, antidepressants, and antioxidants, have been prescribed to improve the oral symptoms of BMS. Venlafaxine or duloxetine was administered to patients who had symptomatic improvement of 50\% or less after taking more than two types of medications over a long period of follow-up (more than 6 months).

\section{Comprehensive Clinical Examinations}

Comprehensive clinical examination procedures included an oral examination, interview, radiographic examination (panoramic view), measurement of the whole salivary flow rate, laboratory test, a simplified psychological evaluation (Symptom Checklist-90-Revision, SCL-90-R), and a BMS questionnaire as described in our previous study [23]. The questionnaire was used to evaluate the clinical characteristics and subjective oral symptoms of patients. The intensities of oral symptoms were measured using a visual analog scale (VAS, 0-10 cm, with 10 being the worst possible). All evaluation procedures were performed by one doctor (HSK). This study was approved by the Institutional Review Board of the Seoul National University Dental Hospital (IRB no. \#ERI18006). The IRB authorized the exemption of informed consent from the patients.

\section{Venlafaxine and Duloxetine Administration}

Venlafaxine or duloxetine were administered at a minimum dosage (venlafaxine, 37.5-75.0 mg/day; duloxetine, $30 \mathrm{mg} /$ day) for 4 weeks. Medications used prior to venlafaxine and duloxetine administration were continued if needed. The patients were instructed to discontinue the venlafaxine or duloxetine if the patients had intolerable side effects. The intensities of oral symptoms were assessed at every visit, and the intensities of oral symptoms after venlafaxine or duloxetine administration were compared with those before administration and at baseline.

\section{RESULTS}

\section{Clinical Characteristics}

Twelve refractory BMS patients were included in this 


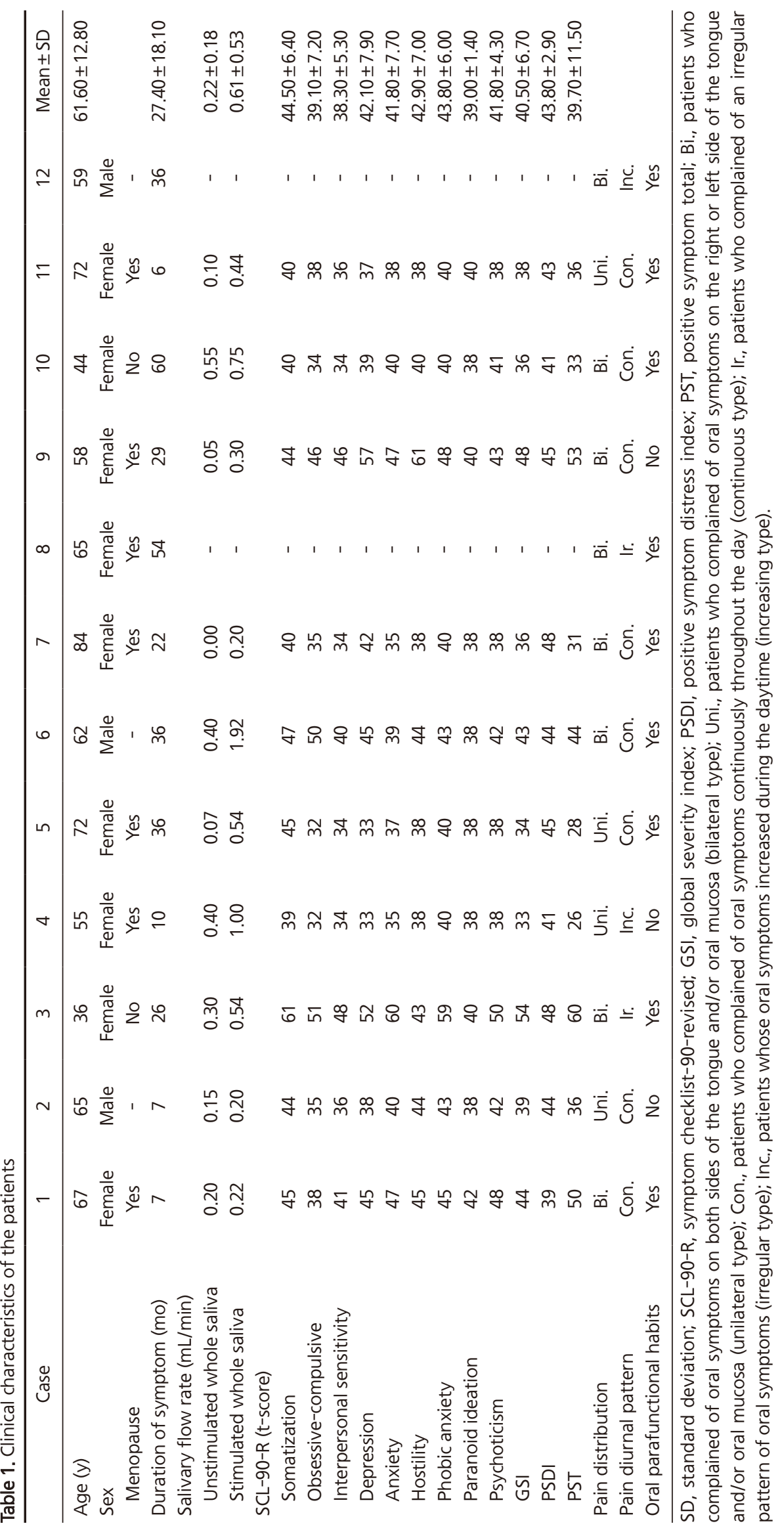


study and comprised three males and nine females. Of the nine female patients, two patients were premenopausal women. The clinical characteristics of the patients are shown in Table 1. The mean ( \pm standard deviation, SD) age of the subjects was $61.6 \pm 12.8$ years. Almost patients were middle-aged or elderly, and only one patient (case 3) was younger than 40 years old. The mean $( \pm S D)$ duration of symptoms was $27.4 \pm 18.1$ months (range, 6-60 months). The whole salivary flow rate was not measured in two patients (cases 8 and 12). The mean $( \pm \mathrm{SD})$ unstimulated and stimulated whole salivary flow rates in 10 patients were $0.22 \pm 0.18$ $\mathrm{mL} / \mathrm{min}$ and $0.61 \pm 0.53 \mathrm{~mL} / \mathrm{min}$, respectively. The number of patients with reduced salivary output in unstimulated and stimulated whole saliva (unstimulated whole saliva, less than $0.1 \mathrm{~mL} / \mathrm{min}$; stimulated whole saliva, less than $0.7 \mathrm{~mL} /$ min) was three and seven, respectively. Two patients (cases 8 and 12) did not undergo SCL-90-R evaluation. The mean t-scores in all symptom dimension and global indices of 10 patients was within the normal range (t-score $<60$ ). In only two cases, the t-score was 60 or above in at least one item (somatization, anxiety, and positive symptom total in case 3; hostility in case 9). In terms of pain distribution, eight patients complained of oral symptoms on both sides of the tongue and/or oral mucosa (bilateral type), and the rest (four patients) complained of oral symptoms on the right or left side of the tongue and/or oral mucosa (unilateral type). With regard to the diurnal pattern of pain, the majority of patients (eight patients) complained of oral symptoms continuously throughout the day (continuous type), and the rest of the patients reported that the oral symptoms were irregular (irregular type, two patients) or increased during the daytime (increasing type, two patients).

\section{Previous Treatment History}

The mean $( \pm \mathrm{SD})$ duration of previous treatments was 129.6 \pm 104.2 weeks (range, 28-344 weeks). Clonazepam, alpha-lipoic acid, gabapentin, and nortriptyline were used as previous treatments for BMS. Most patients were prescribed one or two medications every visit, and up to three medications were prescribed in two cases (case 8, clonazepam, gabapentin, and nortriptyline; case 12, clonazepam, alpha-lipoic acid, and gabapentin). In one case (case 5), she received only one medication at a time. Clonazepam was

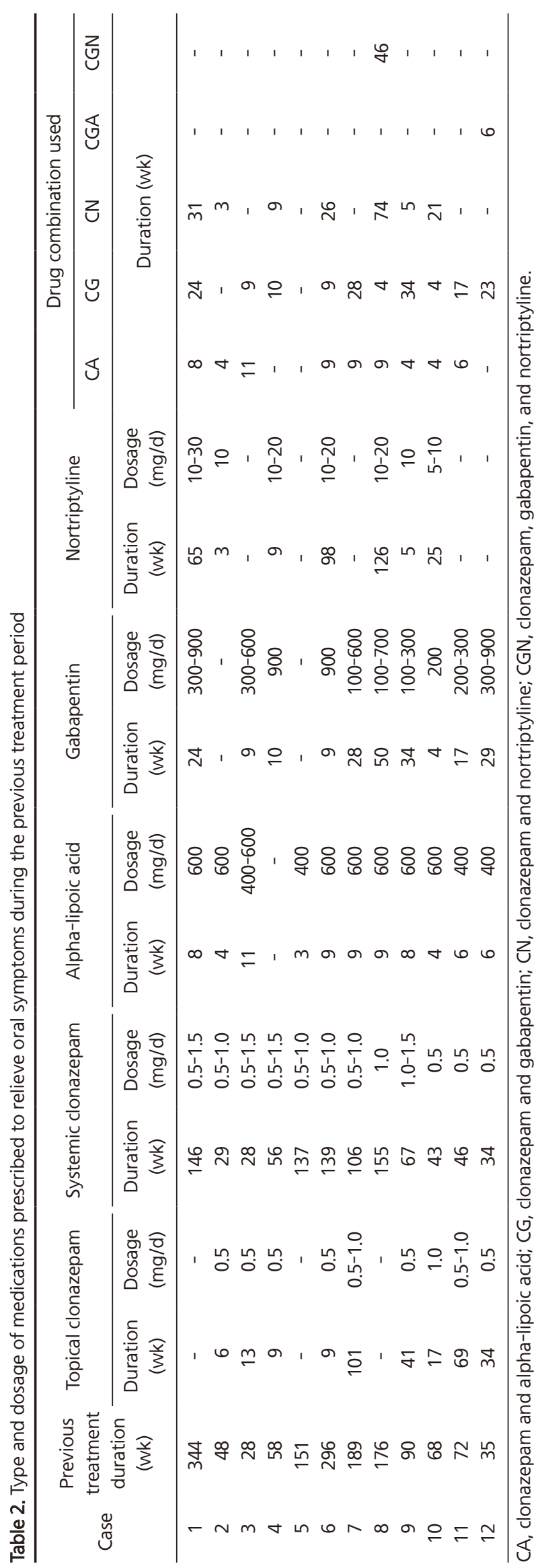


administered to all patients, and was the first selected medication in 11 patients. Only one patient (case 9) was initially prescribed alpha-lipoic acid because the patient had been prescribed clonazepam by another doctor, and the drug was ineffective. Clonazepam was predominantly administered orally, but topical administration was also applied to nine patients. Alpha-lipoic acid was the second most commonly administered medication (11 patients). In most cases alphalipoic acid was prescribed in combination with other medications (mainly clonazepam). In only two cases (cases 5 and 9), alpha-lipoic acid was prescribed alone. Gabapentin was administered to 10 patients, and always prescribed in combination with other medications (mainly clonazepam). Seven patients received nortriptyline. It was prescribed in combination with other medications (mainly clonazepam) in all patients, and four patients were also prescribed nortriptyline alone (Table 2).

\section{Treatment Outcomes of Venlafaxine and Duloxetine}

Four patients were prescribed venlafaxine, and nine patients were prescribed duloxetine. One patient (case 4) was prescribed both medications in turn. Eight patients continued the medications used before venlafaxine and duloxetine administration. Six patients (cases 4, 6, 9, 10, 11, and 12) continued clonazepam, and two patients (cases 7 and 8) continued clonazepam and gabapentin (Table 3).

of the four patients who were prescribed venlafaxine, three patients did not show any improvement of symptoms and reported side effects (insomnia, drowsiness, and headache). Two of them discontinued venlafaxine due to intolerable side effects (insomnia and headache). Of the nine patients who were prescribed duloxetine, seven patients did not show any improvement of symptoms. Three of them reported side effects (insomnia, constipation, dizziness, and nausea). One of the three patients had only mild side effect, and the other two patients discontinued duloxetine due to intolerable side effects. In the other four patients, duloxetine was well tolerated, but failed to relieve symptoms (Table 3).

Venlafaxine or duloxetine only provided relief of oral symptoms in two patients (cases 4 and 8). In case 4, venlafaxine treatment was initiated at a dosage of $37.5 \mathrm{mg} /$ day (once a day at bedtime) for 4 weeks. The patient reported that the symptoms improved gradually, and the VAS score of oral burning sensation also decreased from 5 to 2. The patient continued to receive treatment for the following 4 months. After 5 months of treatment, the patient complained of some side effects (insomnia and xerostomia), so duloxetine treatment was then started at a dosage of 30 $\mathrm{mg} /$ day (once a day, following breakfast to prevent insomnia) for 4 weeks. The patient reported greater improvement in the oral symptoms than when taking venlafaxine and no side effects. The duloxetine treatment was continued because the symptoms worsened after discontinuing the medication. During long-term follow-up (more than 2 years), the patient's BMS symptoms were well controlled through duloxetine administration, and no side effects were reported.

In case 8 , duloxetine treatment was initiated at a dosage of $30 \mathrm{mg} /$ day (once a day, following breakfast) for 4 weeks.

Table 3. Responses to venlafaxine or duloxetine

\begin{tabular}{|c|c|c|c|c|c|c|}
\hline Case & Medication & Discontinuation & $\begin{array}{l}\text { Dosage } \\
(\mathrm{mg} / \mathrm{d})\end{array}$ & Other drugs combined & $\begin{array}{l}\text { Drug response } \\
\text { reported by patients }\end{array}$ & Side effect \\
\hline 1 & Venlafaxine & Yes (about after 2 wk) & 37.5 & None & Ineffective & Yes (insomnia) \\
\hline 2 & Venlafaxine & No & $37.5-75.0$ & None & Ineffective & Yes (drowsiness) \\
\hline 3 & Venlafaxine & Yes (about after 2 wk) & 37.5 & None & Ineffective & Yes (insomnia, headache) \\
\hline \multirow[t]{2}{*}{4} & Venlafaxine & No & 37.5 & Clonazepam & Effective & Yes (insomnia, xerostomia) \\
\hline & Duloxetine & No & 30.0 & Clonazepam & Effective & None \\
\hline 5 & Duloxetine & Yes (about after 2 wk) & 30.0 & None & Ineffective & Yes (insomnia, constipation) \\
\hline 6 & Duloxetine & No & 30.0 & Clonazepam & Ineffective & Yes (dizziness) \\
\hline 7 & Duloxetine & No & 30.0 & Clonazepam, gabapentin & Ineffective & None \\
\hline 8 & Duloxetine & No & 30.0 & Clonazepam, gabapentin & Somewhat effective & None \\
\hline 9 & Duloxetine & Yes (about after 2 wk) & 30.0 & Clonazepam & Ineffective & Yes (nausea) \\
\hline 10 & Duloxetine & No & 30.0 & Clonazepam & Ineffective & None \\
\hline 11 & Duloxetine & No & 30.0 & Clonazepam & Ineffective & None \\
\hline 12 & Duloxetine & No & 30.0 & Clonazepam & Ineffective & None \\
\hline
\end{tabular}


The patient reported that the symptoms were slightly improved and there was a marked reduction in the frequency of pain rather than intensity of pain. The VAS score of oral burning sensation decreased slightly from 4 to 3. During long-term follow-up (more than 1.5 years), the patient's BMS symptoms remained partially reduced, and no side effects were reported.

The mean VAS scores at baseline and before and after the administration of venlafaxine or duloxetine are shown in Fig. 1. The mean VAS scores of oral symptoms in all patients did not show any significant changes except for with the aching type of pain during long-term follow-up. The same results were obtained for non-responders to venlafaxine or duloxetine. The mean VAS scores of oral symptoms in responders to venlafaxine or duloxetine treatment were markedly decreased after treatment.

\section{DISCUSSION}

In this study, we investigated the result of venlafaxine or duloxetine administration in twelve refractory BMS patients who were resistant to clonazepam, alpha-lipoic acid, gabapentin, and nortriptyline therapy. Our findings indicate that venlafaxine and duloxetine may only relieve oral symptoms in a minority of refractory BMS patients.

The descending pain modulation pathway can facilitate or inhibit nociceptive inputs at the spinal level [24], and is mediated by monoamine neurotransmitters, such as serotonin and norepinephrine [25]. These modulatory effects provide a protective mechanism against pain chronicity, and it is believed that the imbalance between facilitation and inhibition of nociceptive input, especially decreased activity in the descending inhibitory pathway, is involved in the development of central sensitization and chronicity in many clinical pain disorders, including BMS [26,27]. Monoamine reuptake inhibitors block the neuronal reuptake of monoamine neurotransmitters and enhance the descending inhibitory pathway $[28,29]$. Because of these pharmacological actions, several monoamine reuptake inhibitors TCAs, selective serotonin reuptake inhibitors (SSRIs), and SNRIs - have been proposed as the first-line therapy for chronic neuropathic pain. In particular, SNRIs inhibit both serotonin and norepinephrine reuptake and have low affinity for other receptors [30,31]. Therefore, SNRIs are believed to have higher analgesic effects and less side effects compared to older antidepressants, such as TCAs and SSRIs.

In the management of BMS, medications targeting the descending inhibitory pathway have also been frequently used. Clonazepam mainly enhances the serotonergic descending inhibition pathway [32,33], and TCAs inhibit both serotonin and norepinephrine reuptake [12]. It has been suggested that acting on either monoaminergic pathway alone is less effective for the production of analgesic effects compared to acting on both simultaneously [34-36]. SNRIs are more relatively balanced reuptake inhibitors of both serotonin and norepinephrine compared to TCAs, and
A

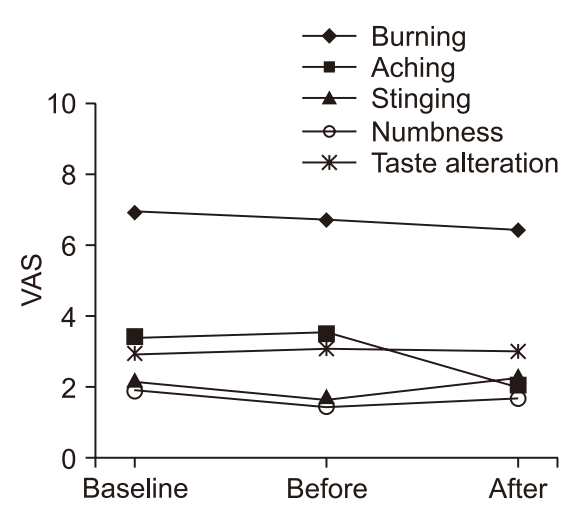

B

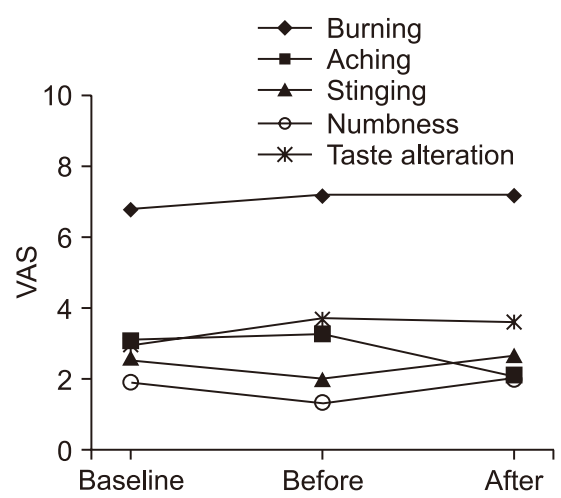

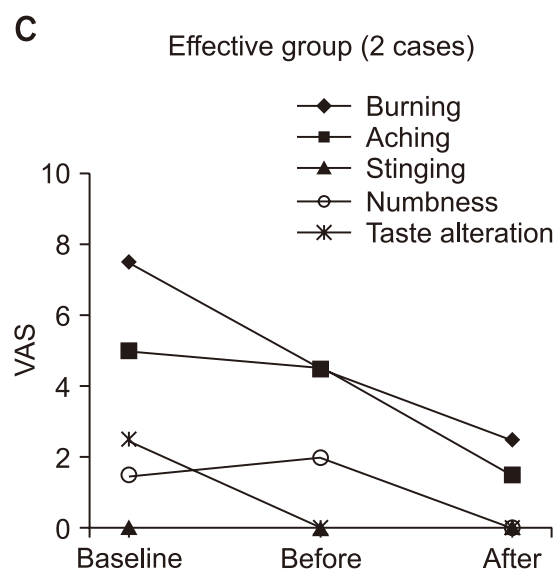

Fig. 1. Comparison of visual analog scale (VAS) scores of oral symptoms at baseline, and before and after venlafaxine or duloxetine administration in (A) all patients, (B) non-responders to venlafaxine or duloxetine, and (C) responders to venlafaxine or duloxetine. Baseline, the first visit to the clinic; before, the visit when the patient was first prescribed venlafaxine or duloxetine; after, the visit 2-4 weeks after the patient was prescribed venlafaxine or duloxetine. 
duloxetine has a similar affinity to both norepinephrine and serotonin transporters than venlafaxine [31]. Indeed, one participant (case 4) who was prescribed both medications in turn reported greater pain relief after duloxetine administration than venlafaxine. Based on these findings, it can be assumed that SNRIs (venlafaxine and duloxetine) may be effective for the management of refractory BMS patients who do not respond to other neuropathic pain medications. In fact, two single case reports and one observational study with small number of subjects have reported the effects of SNRIs on BMS patients [19-21].

However, in contrast to findings from previous studies, our data showed that the majority of refractory BMS patients do not respond to venlafaxine or duloxetine therapy. There are several possible explanations for the insufficient efficacy of venlafaxine and duloxetine in this study. First, the prescription dose of venlafaxine and duloxetine may not be sufficient to give symptom relief. Duloxetine tends to have better analgesic effects at higher doses, and the recommended dose of duloxetine is generally $60 \mathrm{mg}$ once daily [15]. In two single case reports, $60 \mathrm{mg} /$ day of duloxetine was effective in refractory BMS patients [20,21]. Venlafaxine has been shown to be effective in the treatment of neuropathic pain at high doses (higher than $150 \mathrm{mg} /$ day), since at low doses (lower than $150 \mathrm{mg} /$ day), it inhibits serotonin reuptake but not norepinephrine reuptake $[17,18]$. In addition, one previous study reported that high dose of venlafaxine (300 mg/day) combined with clonazepam may be effective in refractory BMS patients [19]. In our study, the daily doses of venlafaxine and duloxetine were $37.5 \mathrm{mg}$ (75 $\mathrm{mg}$ in one case) and $30 \mathrm{mg}$, respectively. Second, the treatment period was relatively short, about and less than 4 weeks. Although venlafaxine and duloxetine may exert their analgesic effects within a short period of time [3739], a long-term follow-up is necessary to accurately assess the analgesic effects of these medications. Therefore, a longer observation period and increase in the prescription dosage are needed to confirm the efficacy of both medications. However, this was difficult since the majority of participants in our study who were refractory to many neuropathic medications complained of intolerable side effects to venlafaxine and duloxetine.

In addition to the observation period and prescription dosage of venlafaxine and duloxetine, the complexity of BMS pathophysiology may affect the efficacy of these medications. Various neuropathic mechanisms are implicated in BMS pathophysiology as well as dysfunction of the descending inhibitory pathway. Therefore, the unique pharmacological properties of SNRIs did not necessarily indicate that SNRIs have a greater analgesic effect than other neuropathic pain medications. Previous studies reported that venlafaxine had no superior efficacy for the relief of neuropathic pain compared to other medications $[37,39,40]$.

In this study, only two patients (cases 4 and 8 ) showed a marked improvement in oral symptoms after venlafaxine or duloxetine therapy. However, since these patients continued the medication (clonazepam and gabapentin) used before venlafaxine and duloxetine administration, the therapeutic effects of venlafaxine and duloxetine may be a combined effect of SNRIs and other medications (clonazepam and gabapentin), and not due to SNRIs alone. Interestingly, one patient who respond to venlafaxine had also been prescribed the clonazepam, which is consistent with one previous study reporting the efficacy of combination therapy with venlafaxine and clonazepam [19]. We could not find any differences in the clinical characteristics between the responders and non-responders to venlafaxine and duloxetine, though these results could be due to the small number of participants. Identifying the relationship between the clinical characteristics and outcome of the medication therapy may be helpful in selecting the appropriate medications, and enables clinicians to provide personalized treatment for individual patients. Further studies are needed to determine the potential clinical factors that could predict the efficacy of venlafaxine and duloxetine.

This study has several limitations. It is a retrospective observational study with a small number of patients. Different medications were prescribed for different periods before venlafaxine and duloxetine administration, and we could not able to control the use of other medications while taking SNRIs. These factors made it difficult to accurately evaluate the efficacy of SNRIs. Therefore, further large-scale randomized controlled trials are needed to assess the efficacy of SNRIs and to compare with other medications to identify their relative benefits in BMS.

In conclusions, venlafaxine and duloxetine may only 
relieve oral symptoms in a minority of refractory BMS patients. Nevertheless, given the limited treatment options of BMS, venlafaxine and duloxetine should be considered as one of the treatment options for BMS. Further large-scale studies are necessary to determine the clinical usefulness of venlafaxine and duloxetine in the management of BMS.

\section{CONFLICT OF INTEREST}

No potential conflict of interest relevant to this article was reported.

\section{ORCID}

\author{
Moon-Jong Kim \\ https://orcid.org/0000-0002-9518-0176 \\ Hong-Seop Kho \\ https://orcid.org/0000-0001-9960-9892
}

\section{REFERENCES}

1. Scala A, Checchi L, Montevecchi M, Marini I, Giamberardino MA. Update on burning mouth syndrome: overview and patient management. Crit Rev Oral Biol Med 2003;14:275-291.

2. Klasser GD, Grushka M, Su N. Burning mouth syndrome. Oral Maxillofac Surg Clin North Am 2016;28:381-396.

3. Jääskeläinen SK. Pathophysiology of primary burning mouth syndrome. Clin Neurophysiol 2012;123:71-77.

4. Jääskeläinen SK. Is burning mouth syndrome a neuropathic pain condition? Pain 2018;159:610-613.

5. Woda A, Navez ML, Picard P, Gremeau C, Pichard-Leandri E. A possible therapeutic solution for stomatodynia (burning mouth syndrome). J Orofac Pain 1998;12:272-278.

6. Gremeau-Richard C, Woda A, Navez ML, et al. Topical clonazepam in stomatodynia: a randomised placebo-controlled study. Pain 2004;108:51-57.

7. Amos K, Yeoh SC, Farah CS. Combined topical and systemic clonazepam therapy for the management of burning mouth syndrome: a retrospective pilot study. J Orofac Pain 2011;25:125130.

8. Cui Y, Xu H, Chen FM, et al. Efficacy evaluation of clonazepam for symptom remission in burning mouth syndrome: a metaanalysis. Oral Dis 2016;22:503-511.

9. Liu YF, Kim Y, Yoo T, Han P, Inman JC. Burning mouth syndrome: a systematic review of treatments. Oral Dis 2018;24:325344.

10. López-D’alessandro E, Escovich L. Combination of alpha lipoic acid and gabapentin, its efficacy in the treatment of Burning Mouth Syndrome: a randomized, double-blind, placebo con- trolled trial. Med Oral Patol Oral Cir Bucal 2011;16:e635-e640.

11. Mott AE, Grushka M, Sessle BJ. Diagnosis and management of taste disorders and burning mouth syndrome. Dent Clin North Am 1993;37:33-71.

12. Fenelon M, Quinque E, Arrive E, Catros S, Fricain JC. Painrelieving effects of clonazepam and amitriptyline in burning mouth syndrome: a retrospective study. Int J Oral Maxillofac Surg 2017;46:1505-1511.

13. Sardella A, Lodi G, Demarosi F, Bez C, Cassano S, Carrassi A. Burning mouth syndrome: a retrospective study investigating spontaneous remission and response to treatments. Oral Dis 2006;12:152-155.

14. Rodríguez-de Rivera-Campillo E, López-López J. Evaluation of the response to treatment and clinical evolution in patients with burning mouth syndrome. Med Oral Patol Oral Cir Bucal 2013;18:e403-e410.

15. Bellingham GA, Peng PW. Duloxetine: a review of its pharmacology and use in chronic pain management. Reg Anesth Pain Med 2010;35:294-303.

16. Lunn MP, Hughes RA, Wiffen PJ. Duloxetine for treating painful neuropathy, chronic pain or fibromyalgia. Cochrane Database Syst Rev 2014;(1):CD007115.

17. Trouvin AP, Perrot S, Lloret-Linares C. Efficacy of venlafaxine in neuropathic pain: a narrative review of optimized treatment. Clin Ther 2017;39:1104-1122.

18. Aiyer R, Barkin RL, Bhatia A. Treatment of neuropathic pain with venlafaxine: a systematic review. Pain Med 2017;18:1999-2012.

19. Mitsikostas DD, Ljubisavljevic S, Deligianni CI. Refractory burning mouth syndrome: clinical and paraclinical evaluation, comorbiities, treatment and outcome. J Headache Pain 2017;18:40.

20. Mignogna MD, Adamo D, Schiavone V, Ravel MG, Fortuna G. Burning mouth syndrome responsive to duloxetine: a case report. Pain Med 2011;12:466-469.

21. Kim YD, Lee JH, Shim JH. Duloxetine in the treatment of burning mouth syndrome refractory to conventional treatment: a case report. J Int Med Res 2014;42:879-883.

22. Ito M, Tokura T, Yoshida K, et al. Five patients with burning mouth syndrome in whom an antidepressant (serotonin-noradrenaline reuptake inhibitor) was not effective, but pregabalin markedly relieved pain. Clin Neuropharmacol 2015;38:158-161.

23. Kim MJ, Kim J, Kho HS. Comparison between burning mouth syndrome patients with and without psychological problems. Int J Oral Maxillofac Surg 2018;47:879-887.

24. Kwon M, Altin M, Duenas H, Alev L. The role of descending inhibitory pathways on chronic pain modulation and clinical implications. Pain Pract 2014;14:656-667.

25. Millan MJ. Descending control of pain. Prog Neurobiol 2002; 66:355-474.

26. Albuquerque RJ, de Leeuw R, Carlson CR, Okeson JP, Miller CS, Andersen AH. Cerebral activation during thermal stimulation of patients who have burning mouth disorder: an fMRI study. Pain 2006;122:223-234.

27. Ossipov MH, Morimura K, Porreca F. Descending pain modulation and chronification of pain. Curr Opin Support Palliat Care 2014;8:143-151. 
28. Gillman PK. Tricyclic antidepressant pharmacology and therapeutic drug interactions updated. Br J Pharmacol 2007;151:737748.

29. Argoff C. Mechanisms of pain transmission and pharmacologic management. Curr Med Res Opin 2011;27:2019-2031.

30. Wong DT, Bymaster FP, Mayle DA, Reid LR, Krushinski JH, Robertson DW. LY248686, a new inhibitor of serotonin and norepinephrine uptake. Neuropsychopharmacology 1993;8:23-33.

31. Bymaster FP, Dreshfield-Ahmad LJ, Threlkeld PG, et al. Comparative affinity of duloxetine and venlafaxine for serotonin and norepinephrine transporters in vitro and in vivo, human serotonin receptor subtypes, and other neuronal receptors. Neuropsychopharmacology 2001;25:871-880.

32. Grushka M, Epstein J, Mott A. An open-label, dose escalation pilot study of the effect of clonazepam in burning mouth syndrome. Oral Surg Oral Med Oral Pathol Oral Radiol Endod 1998;86:557-561.

33. Heckmann SM, Kirchner E, Grushka M, Wichmann MG, Hummel T. A double-blind study on clonazepam in patients with burning mouth syndrome. Laryngoscope 2012;122:813-816.

34. Marchand F, Alloui A, Chapuy E, et al. Evidence for a monoamine mediated, opioid-independent, antihyperalgesic effect of venlafaxine, a non-tricyclic antidepressant, in a neurogenic pain model in rats. Pain 2003;103:229-235.

35. Iyengar S, Webster AA, Hemrick-Luecke SK, Xu JY, Simmons RM. Efficacy of duloxetine, a potent and balanced serotoninnorepinephrine reuptake inhibitor in persistent pain models in rats. J Pharmacol Exp Ther 2004;311:576-584.

36. Lee YC, Chen PP. A review of SSRIs and SNRIs in neuropathic pain. Expert Opin Pharmacother 2010;11:2813-2825.

37. Sindrup SH, Bach FW, Madsen C, Gram LF, Jensen TS. Venlafaxine versus imipramine in painful polyneuropathy: a randomized, controlled trial. Neurology 2003;60:1284-1289.

38. Fishbain D, Berman K, Kajdasz DK. Duloxetine for neuropathic pain based on recent clinical trials. Curr Pain Headache Rep 2006;10:199-204.

39. Razazian N, Baziyar M, Moradian N, Afshari D, Bostani A, Mahmoodi M. Evaluation of the efficacy and safety of pregabalin, venlafaxine, and carbamazepine in patients with painful diabetic peripheral neuropathy. A randomized, double-blind trial. Neurosciences (Riyadh) 2014;19:192-198.

40. Amr YM, Yousef AA. Evaluation of efficacy of the perioperative administration of Venlafaxine or gabapentin on acute and chronic postmastectomy pain. Clin J Pain 2010;26:381-385. 